\title{
Optic nerve vasomotor effects of topical apraclonidine hydrochloride
}

\author{
Selim Orgül, David R Bacon, E Michael Van Buskirk, George A Cioffi
}

\begin{abstract}
Aims-To examine, in vivo, the anterior optic nerve vasomotor effects of chronic apraclonidine hydrochloride in rabbits.

Methods-After local treatment in one randomly chosen eye with apraclonidine hydrochloride $0.5 \%$ over 21 days, the microvasculature of the optic nerve was examined in five rabbits using an intraluminal microvascular corrosion casting technique. The investigators were masked as to which eye was treated. The vasoconstriction near the branching point of arterioles supplying the optic nerve was calculated as a percentage of the downstream vessel calibre. An average constriction was calculated and compared between the treated and the contralateral, untreated, eyes by means of a two tailed $t$ test for paired variables. Constriction values of a total of 72 arterioles supplying the optic nerve were obtained for the five rabbits.
\end{abstract}

Results-The average constriction in the treated and the control eyes was comparable $(p=0.96)$.

Conclusion-Chronic administration of apraclonidine hydrochloride $0.5 \%$ produces no observable optic nerve vasomotor effects in the rabbit eye.

(Br $\mathcal{F}$ Ophthalmol 1996; 80: 82-84)

Apraclonidine hydrochloride is an $\alpha_{2}$ adrenergic agonist which is a very efficient ocular hypotensive agent in subjects with normal or increased intraocular pressure. ${ }^{1-5}$ Prevailing studies suggest that apraclonidine hydrochloride may be a reasonable choice for use in conjunction with $\beta$ blocker therapy. ${ }^{6}$ However, many clinical observations lend credence to the potential role of microcirculatory changes in glaucoma, ${ }^{7}$ and there is concern whether some topical antiglaucomatous drugs, although effective in lowering intraocular pressure, could have adverse vascular effects. ${ }^{8}$ Apraclonidine hydrochloride manifests potent vasoconstrictive activity in anterior segment tissues of the eye. ${ }^{910}$ However, optic nerve blood flow seems not to be affected by single dose topical or intravenous administration of apraclonidine hydrochloride in monkeys, rabbits, or cats. ${ }^{11-13}$

The present study was designed to evaluate, in vivo, whether chronic topical administration of apraclonidine hydrochloride may induce vascular constrictions in the anterior optic nerve vascular bed in an animal model.
Materials and methods

Five adult New Zealand white rabbits of both sexes, weighing $\geqslant 2.5 \mathrm{~kg}$, were assigned to 21 days of topical ocular treatment with apraclonidine hydrochloride $0.5 \%$. The eyedrops were administered once a day in one randomly chosen eye. All experiments conformed to the Association for Research in Vision and Ophthalmology resolution on the use of animals in research.

The microvasculature of the optic nerve was examined with the intraluminal microvascular corrosion casting technique described in detail elsewhere. ${ }^{14}$ In brief, the castings of the ocular vasculature were obtained under controlled physiological conditions. The rabbits were anaesthetised with intravenous sodium pentobarbitone. Mechanical ventilation was provided by a Harvard small animal respirator. Systemic blood pressure, blood gases, and rectal body temperature were maintained stable. Arterial blood gases were drawn from an indwelling aortic catheter immediately before injection. Batson's no 17 methylmethacrylate injection media, modified to reduce the viscosity to approximately 11 centipoise, was injected into the superior circulation through ascending branches of the aorta. The injection pressure was maintained at approximately 100 $\mathrm{mm} \mathrm{Hg}$ for 15 minutes, until the plastic began to polymerise and arrest flow in the vascular system. Two hours after injection, the eyes were enucleated, stored overnight in warm formalin to complete the polymerisation, and corroded in $6 \mathrm{~mol} / 1$ potassium hydroxide. The resulting plastic vascular castings were rinsed in running water and air dried. Whole globe vascular castings were hemisected at the equator and the posterior segments were mounted on aluminium stubs, sputter coated with gold palladium, and examined with a scanning electron microscope (Etec Autoscan).

The optic nerve microvasculature in rabbits is primarily supplied by the short posterior ciliary arteries via an arterial loop encircling the optic nerve. ${ }^{15}$ Direct arteriolar branches from the short posterior ciliary arteries or branches from the arterial circle which penetrate and supply the optic nerve normally display focal zones of relative constriction near their branching point from the proximal arterial supply, compared with downstream vessel calibre. ${ }^{16}$ The primary and secondary arteriolar branches from the arterial circle were measured at these constriction zones near the branching point and $50 \mu \mathrm{m}$ distal to the end of the constriction zone (Fig 1). Because of the large variation in vascular diameter as well as the statistical 


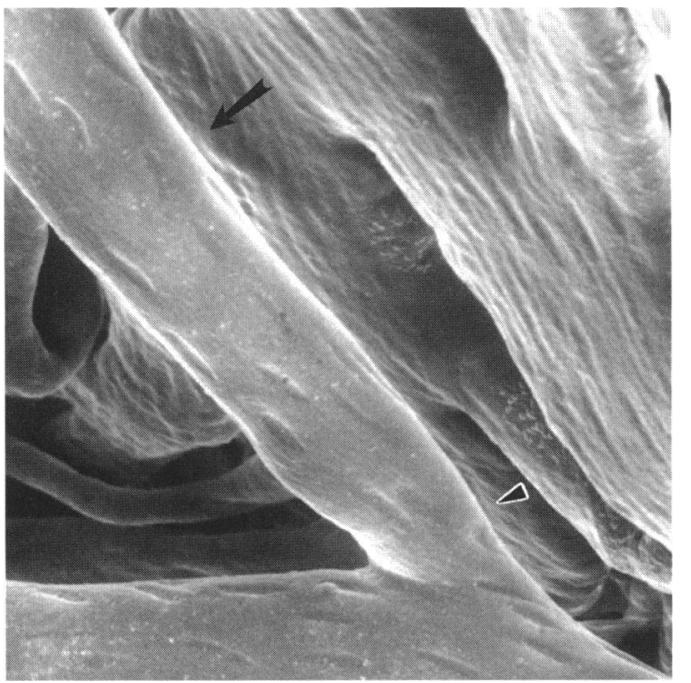

Figure 1 Example of an arterial vessel which supplies the optic nerve and displays a focal zone of relative constriction near the branching point (arrowhead), compared with downstream vessel calibre (arrow).

variability of individual vascular responses, earlier studies did not show significant differences in actual vessel diameter after pharmacological manipulations. ${ }^{101417}$ For this reason, the present analysis was confined to the assessment of the relative branch point constrictions. The relative amount of branch point constriction at the focal zones near the branching point was expressed as a percentage constriction relative to the distal diameter (100X (distal diameter-constricted diameter/distal diameter)). Analysing specific vessels alone with corrosion casting technique provides very limited functional information for the optic nerve microvasculature. Only vessels providing distinct measurement sites at their branching point and $50 \mu \mathrm{m}$ distal from this point can be included in the analysis. Owing to the complex anatomy of the anterior optic nerve microvasculature, this restriction limits significantly the number of the vessels which can be included in the analysis. For the present analysis, arteriolar branches from the arterial circle around the optic nerve, just adjacent to the posterior portion of the choroidal vasculature, which penetrate and supply the optic nerve were included. ${ }^{15}$ Because often the short length of primary branches from the arterial circle precludes a measurement $50 \mu \mathrm{m}$ distal from the branching point, secondary branches were included as well. During the measurements, the investigators were masked to the rabbit and to the treatment. The measurements of arteriolar constriction as a percentage of the downstream vessel calibre were pooled for each eye and an average constriction value was calculated for each eye. The average constriction was compared in the treated eyes and the contralateral, untreated eyes by means of a two tailed $t$ test for paired variables. More over, because the varying number of vessels assessed in the eyes causes a differential contribution of the animals to the data, a common maximum number of vessels per eye (selected randomly for the eyes providing more vessels) was used to analyse the difference between the treated and untreated eyes as described above.
Table 1 Average relative arteriolar branch point constrictions (\%)

\begin{tabular}{lll}
\hline Rabbit & $\begin{array}{l}\text { Treated eye } \\
\text { (number of vessels) }\end{array}$ & $\begin{array}{l}\text { Control eye } \\
\text { (number of vessels) }\end{array}$ \\
\hline 1 & $18 \cdot 20(9)$ & $22.93(7)$ \\
2 & $23.98(11)$ & $25 \cdot 61(14)$ \\
3 & $28 \cdot 19(4)$ & $17 \cdot 21(8)$ \\
4 & $21.35(5)$ & $24 \cdot 88(5)$ \\
5 & $22 \cdot 68(5)$ & $24 \cdot 48(4)$ \\
\hline
\end{tabular}

The intraocular pressure was measured three times with a pneumotonometer in both eyes after general anaesthesia before intraluminal microvascular corrosion casting. The pneumotonometer was calibrated before intraocular pressure measurements. An average value of intraocular pressure was calculated for each eye. The intraocular pressure in the treated eyes and the contralateral, untreated eyes was compared by means of a two tailed $t$ test for paired variables.

\section{Results}

Constriction values of a total of 72 arterioles supplying the optic nerve ranged from $0 \%$ to $42.86 \%$ (Fig 1). The average (SD) number of vessels analysed per eye was 7 (3) vessels (range 4-14 vessels per eye). The average arteriolar constriction values are shown in Table 1. The mean (SD) average constriction for the treated eyes was $22 \cdot 88 \%(3 \cdot 66 \%)$. The mean (SD) average constriction for the contralateral, untreated eyes was $23.02 \%$ (3.31\%). No statistically significant differences were found between the treated eyes and the contralateral, untreated eyes (mean difference $0 \cdot 14 \% ; 95 \%$ confidence interval (CI) of the difference -7.74 to $8.02 ; p=0.96)$. After selecting four vessels (maximum common number of vessels per eye), the mean (SD) average constriction was $21 \cdot 90 \%(4 \cdot 15 \%)$ and $21 \cdot 85 \%(3 \cdot 85 \%)$ for the treated and untreated eyes, respectively. These values were comparable as well (mean difference $0.05 \% ; 95 \%$ CI of the difference -6.57 to $6 \cdot 67 ; p=0 \cdot 98$ ).

The intraocular pressure was comparable in the treated $(11.47(1.17) \mathrm{mm} \mathrm{Hg})$ and untreated $(12.23 \quad(3.07) \mathrm{mm} \quad \mathrm{Hg})$ eyes $\mathrm{p}=0 \cdot 60)$.

\section{Discussion}

The current study suggests that topical apraclonidine hydrochloride $0.5 \%$ does not produce vasomotor effects in the optic nerve of the rabbit eye. No statistically significant differences between treated and untreated eyes were found.

Preservation of the visual field is the main goal of the antiglaucomatous therapy. It is theoretically possible that adverse vascular effects of some topical drugs might tend to neutralise the beneficial effect of a reduced intraocular pressure on the preservation of the visual field. Apraclonidine hydrochloride manifests a potent vasoconstrictive activity in anterior segment tissues. ${ }^{9-12}$ In view of the potential role of microcirculatory changes in glaucoma, ${ }^{7}$ such a mighty vasoconstrictive 
drug may have a deleterious effect on visual field survival in glaucoma patients on long term therapy.

Topical ocular treatment with apraclonidine hydrochloride has been shown to cause substantial constriction in arterioles that supply the ciliary processes of albino rabbits. ${ }^{10}$ This study may have raised concerns about impaired vascular perfusion in glaucoma patients undergoing a long term therapy with apraclonidine hydrochloride. Optic nerve blood flow, however, seems not to be affected by single dose topical or intravenous administration of apraclonidine hydrochloride in monkeys, cats, or rabbits. ${ }^{11-13}$ The present data suggest, furthermore, that a chronic topical administration of apraclonidine hydrochloride $0.5 \%$ does not provoke arteriolar vasoconstriction in the anterior optic nerve vascular bed.

While corrosion casting seems to be an excellent method for studying the anatomy of a microvascular system, the application of this technique to study vasoreactivity is more controversial. Reasonable concerns have arisen about vascular alterations induced by the plastic itself during the injection process. Earlier studies, however, could show that the methacrylate itself has little or no vasoreactivity on microvessels. ${ }^{14}$ Furthermore, it has been demonstrated that drug related vasomotor effects can be induced by $\alpha$ adrenergic agonists in the anterior vascular bed of the optic nerve in albino rabbits after chronic topical ocular treatment and that these effects can be shown by means of intravascular casting technique. ${ }^{16}$ In contrast with the ciliary vessels, the filling of the optic nerve microvasculature is more inconsistent. ${ }^{16}$ For this reason, maintaining the physiological variables constant throughout the surgical procedure is of primary importance. Improving the monitoring of the physiological variables has shown a tremendous improvement in the filling of the optic nerve microvasculature, but also an increase of the arteriolar constrictions. In a consequent study, the robustness of the corrosion casting technique to overcome potential methodological artefacts to demonstrate the known physiological effect of variations of blood gases was analysed. ${ }^{18}$ The latter study demonstrated a high correlation between arteriolar branch point constriction of the vessels supplying the optic nerve and arterial blood gases. In the latter investigation, the average constriction in the optic nerve microvasculature was of approximately $22 \%$ with physiological blood gases. Further studies confirmed similar constriction values in placebo treated eyes. ${ }^{19}$ Therefore, a contralateral effect in the untreated eye with a consequent increase of arteriolar constriction on both sides in the present study is improbable.

In conclusion, chronic topical administration of apraclonidine hydrochloride $0.5 \%$ does not seem to have major adverse effects on the microvasculature of the anterior optic nerve. None the less, the long term safety of this medication with regard to optic nerve perfusion remains to be confirmed in glaucoma patients.

This study was supported in part by the Swiss National Science Foundation, the Florian Verrey Foundation (Switzerland), and by National Institutes of Health grant EYO5231.

1 Jampel HD, Robin AL, Quigley HA, Pollack IP Apraclonidine: a one-week dose-response study. Arch Ophthalmol 1988; 106: 1069-73.

2 Abrams DA, Robin AL, Crandall AS, Caldwell DR, Schnitzer DB, Pollack IP, et al. A limited comparison of apraclonidine's dose response in subjects with normal or 108: $230-7$.

3 Abrams DA, Robin AL, Pollack IP, deFaller JM, DeSanti L. The safety and efficacy of topical $1 \%$ ALO 2145 (p-aminoclonidine hydrochloride) in normal volunteers. Arch Ophthalmol 1987; 105: 1205-7.

4 Nagasubramanian S, Hitchings RA, Demailly P, Chuniaud M, Pannarale MR, Pecori-Giraldi J, et al. Comparison of apraclonidine and timolol in chronic open-angle glaucoma. Ophthalmology 1993; 100: 1318-23.

5 Morrison JC, Robin AL. Adjunctive glaucoma therapy: a comparison of apraclonidine to dipivefrin when added to timolol maleate. Ophthalmology 1989; 96: 3-7.

6 Chacko DM, Camras CB. The potential of $\alpha_{2}$-adrenergic agonists in the medical treatment of glaucoma. Curr Opin Ophthalmol 1994; 5: 76-84.

7 Van Buskirk EM. Glaucomatous optic neuropathy. f Glaucoma 1994; 3 (suppl): S2-4.

8 Crick RP, Newson RB, Shipley MJ, Blackmore $H$ Bulpitt CJ. The progress of the visual field in chronic simple glaucoma and ocular hypertension treated topically with pilocarpine or with timolol. Eye 1990; 4: 563-71.

9 Serdahl CL, Galustian J, Lewis RA. The effects of apraclonidine on conjunctival oxygen tension. Arch apraclonidine on conjunctival

10 Van Buskirk EM. Replication of ciliary vasomotor effects with controlled intravascular corrosion casting. Trans $A m$ Ophthalmol Soc 1989; 87: 125-40.

1 Camras CB. Clinical applications of $\alpha_{2}$-adrenergic agonist in ophthalmology. $\mathcal{F}$ Glaucoma $1995 ; 4$ (Suppl 1): S30-5.

12 Kaufman PL, Gabelt B. $\alpha_{2}$-Adrenergic agonist effects on aqueous humor dynamics. F Glaucoma 1995; 4 (Suppl 1): S8-14.

13 Cioffi GA, Orgül S, Bacon DR, Van Buskirk EM. Acute vasomotor effects in the anterior optic nerve of topical apraclonidine hydrochloride. F Glaucoma 1995; 4 (Supp 1): S15-8.

14 Fahrenbach WH, Bacon DR, Morrison JC, Van Buskirk EM. Controlled vascular corrosion casting of the rabbit eye. F Electron Microsc Technique 1988; 10: 15-26.

15 Sugiyama K, Bacon DR, Morrison JC, Van Buskirk EM. Optic nerve head microvasculature of the rabbit eye. Invest Ophthalmol Vis Sci 1992; 33: 2251-61.

16 Sugiyama K, Bacon Dr, Cioffi GA, Fahrenbach WH, Van Buskirk EM. The effects of phenylephrine on the ciliary body and optic nerve head microvasculature in rabbits. body and optic nerve head mic

17 Van Buskirk EM, Bacon DR, Fahrenbach WH. Ciliary vasoconstriction after topical adrenergic drugs. $A m \mathscr{f}$ Ophthalmol 1990; 109: 511-7.

18 Orgül S, Cioffi GA, Bacon DR, Onda E, Van Buskirk EM Optic nerve vasomotor effects of arterial blood gases. F Glaucoma 1995; 4: 322-6.

19 Orgül S, Mansberger S, Bacon DR, Van Buskirk EM, Cioffi GA. Optic nerve vasomotor effects of topical betaadrenergic antagonists. Am $f$ Ophthalmol 1995; 120: adrenergic 